\title{
Terahertz Switch Utilizing Inorganic Perovskite-Embedded Metasurface
}

\author{
Ri-Hui Xiong ${ }^{1}$, Xiao-Qing Peng ${ }^{2}$ and Jiu-Sheng $L_{i}{ }^{1 *}$ \\ ${ }^{1}$ Centre for THz Research, China Jiliang University, Hangzhou, China, ${ }^{2}$ State Grid Sichuan Electric Power Company, \\ Chengdu, China
}

Various applications of terahertz technology require a large number of various terahertz wave control devices. Yet, high efficient and rapid-response terahertz switch is still a great challenge. Here, we introduce a new scheme, based on inorganic perovskite quantum dot (CsPbBr 3 -QD)-embedded metasurface under different pump laser powers that realize terahertz wave high-speed switching performance. The off-on speed of the presented device achieves $8 \mathrm{MHz}$. This kind of components provides a new idea and a cost-effective functional solution for manipulating the terahertz waves in emerging terahertz devices and systems.

Keywords: terahertz wave switch, metasurface, switching speed, terahertz, inorganic pervoskite

\section{OPEN ACCESS}

Edited by:

Lin Chen,

University of Shanghai for Science and

Technology, China

Reviewed by:

Fei Fan,

Nankai University, China Rajour Tanyi Ako,

RMIT University, Australia

*Correspondence:

Jiu-Sheng Li

lijsh2008@126.com

Specialty section:

This article was submitted to Optics and Photonics,

a section of the journal

Frontiers in Physics

Received: 22 January 2020 Accepted: 09 April 2020

Published: 20 May 2020

Citation:

Xiong R-H, Peng X-Q and Li J-S (2020) Terahertz Switch Utilizing Inorganic Perovskite-Embedded Metasurface. Front. Phys. 8:141.

doi: 10.3389/fphy.2020.00141

\section{INTRODUCTION}

Terahertz technology shows great promise for many applications including sensing, spectroscopy, non-destructive imaging, security monitoring, and wireless communications [1-4]. In particular, wireless communication using terahertz wave has attracted much interest due to large frequency bandwidth and high data transmission speed [5, 6]. These applications require the ability to flexibly manipulate terahertz wave in free space. Over the past several years, many kinds of terahertz wave devices have been reported such as filter [7], polarizer [8], power divider [9], modulator [10], absorber [11], switch [12], etc. As the core device of terahertz wave system, terahertz wave switch has received significant attention. Several techniques have been introduced to implement terahertz wave switch by applying external magnetism, electricity, temperature, and light illumination stimuli [13-17]. However, manipulating terahertz wave to achieve high efficient and rapid switching performance in a device is a great challenge.

Inorganic perovskite quantum dots $\left(\mathrm{CsPbBr}_{3}-\mathrm{QDs}\right)$ have recently gained significant attention in photovoltaic applications demonstrating power conversion efficiencies in solar cells, high charge-carrier mobility [18, 19], and longer diffusion lengths [20]. Under the excitation of optical pump fluences, the perovskite exhibits high light absorption and short carrier recombination lifetime on nanosecond [21, 22]. Using these properties of perovskite, they are combined with subwavelength metasurface structures, and their properties can be enhanced or exploited as an efficient terahertz manipulation device. In this work, we described a method for the active control of terahertz wave transmission using $\mathrm{CsPbBr}_{3}$-QD-embedded metasurface. By using commercially available finite difference frequency domain solver CST Microwave Studio, we obtained the optimized dimensions of the $\mathrm{CsPbr}_{3}$-QD-embedded metasurface. Finally, we experimentally demonstrate the switching phenomenon in the $\mathrm{CsPBr}_{3}-\mathrm{QD}$ embedded metasurface using optical stimuli. The proposed switch approach will be of great significance for practical terahertz applications. 


\section{DESIGN AND ANALYSIS}

Figure 1A illustrates the configuration of the $\mathrm{CsPbr}_{3}-\mathrm{QD}$ embedded metasurface-based terahertz wave switch. The top layer employs the inorganic perovskite QD-embedded metasurface as the geometric cell, and it is printed on a silicon oxide $(100 \mathrm{~nm}) /$ high resistance silicon dielectric substrate with relative permittivity of $\varepsilon_{s i}=11.9$ and a thickness of $200 \mu \mathrm{m}$. The metasurface is made of copper with a conductivity of 5.96 $\times 10^{7} \mathrm{~m} / \mathrm{s}$ and a thickness of $300 \mathrm{~nm}$. The dielectric constant of copper from $0.2 \mathrm{THz}$ to $1.0 \mathrm{THz}$ is $\varepsilon_{\mathrm{cu}}=-7.479 \times 10^{4}+$ $i 2.723 \times 10^{6} \sim-7.345 \times 10^{4}+i 5.338 \times 10^{5}$. The dielectric constant of inorganic perovskite QD perovskite in the frequency band $0.2 \sim 1.0 \mathrm{THz}$ is $\varepsilon_{\mathrm{p}}=9.2$ without laser irradiation [17]. The period of the unit cell is $100 \mu \mathrm{m}$. Optical microscope image of the fabricated $\mathrm{CsPbBr}_{3}$-QD-embedded metasurface is shown in Figure 1B. We use the Drude model to describe the perovskite complex conductivity, which can be expressed by Yettapu et al. [22].

$$
\delta(\omega)=\frac{\varepsilon_{0} \omega_{p}^{2}}{t_{\Gamma}-i \omega}\left(1+\frac{C t_{\Gamma}}{t_{\Gamma}-i \omega}\right)
$$

where $\omega_{p}=\sqrt{n} e^{2} / \varepsilon_{0} m$ is the plasma frequency, $t_{\Gamma}$ is the carrier scattering rate as $t_{\Gamma}=e / m^{*} v, m^{*}$ is the carrier effective mass, $m=0.5\left(m_{e}+m_{h}\right), m_{e}=0.22$, and $m_{h}=0.24$. The $C$ parameter, which represents the degree of carrier localization, may have values between 0 and -1 . Without laser irradiate, we can choose $C=0$. While with laser irradiate, we set $C=-1$. The relationship between the conductivity of perovskite and pump laser radiation can be obtained in Chanana et al. [23].

A commercially available finite difference frequency domain solver software CST Microwave Studio was used to simulate the metasurface structure. We use an adaptive mesh with a size of $\lambda / 10$, where $\lambda$ is the wavelength of incident radiation. The surrounding boundary condition was set as the periodic boundary, and an open boundary condition is set along the direction of the terahertz wave propagation. Figure 2 shows the terahertz transmission spectra of the different parts of our proposed metasurface unit cell. One can see that only the $\mathrm{CsPbBr}_{3}$-QD-embedded metasurface unit cell achieved narrow band resonance effects for enhancing the interaction between the terahertz wave and the metasurface. We also numerically analyze the terahertz transmission properties of the structure with different size parameters $g, s, d$, and $a$. In Figure 3, we can find that these parameters only have a weak influence on the position and width of transmission peak at $0.6 \mathrm{THz}$. After completing the optimization process, the geometrical dimensions of the geometric pattern metasurface have been set as follows: $a$ $=75 \mu \mathrm{m}, g=12 \mu \mathrm{m}, s=12 \mu \mathrm{m}$, and $d=70 \mu \mathrm{m}$.

\section{DEVICE FABRICATION}

The metasurface was fabricated using the conventional photolithography technique. First, positive photoresist was coated on a silicon substrate and prebaked at $105^{\circ} \mathrm{C}$ for $1 \mathrm{~min}$. Then, the mask was aligned and exposed under UV-light. The sample was immersed in the developer solution to remove the exposed part of the photoresist. A 300-nm-thick copper was deposited by thermal evaporation, and the sample was removed in acetone solution to obtain the designed metal pattern. Second, a thin layer of parylene $c$ was deposited by vapor deposition to make the parylene film completely cover the sample, followed by a certain complementary pattern in the photoresist. The parylene film was etched with oxygen plasma, leaving a through hole, in which $\mathrm{Cs} \mathrm{PbBr}_{3}$ perovskite QDs can be deposited. After solution casting and annealing of the desired $\mathrm{CsPbBr}_{3}$ perovskite QD film, once the $\mathrm{CsPbr}_{3}$ perovskite QD film starts to crystallize, usually after $60 \mathrm{~s}$, the parylene film will be layered. Finally, the samples were thoroughly annealed to obtain a high-quality polycrystalline pattern structure.

\section{RESULTS AND DISCUSSION}

A terahertz time domain spectroscopy (THz-TDS) system was used to measure the transmission spectra of the sample
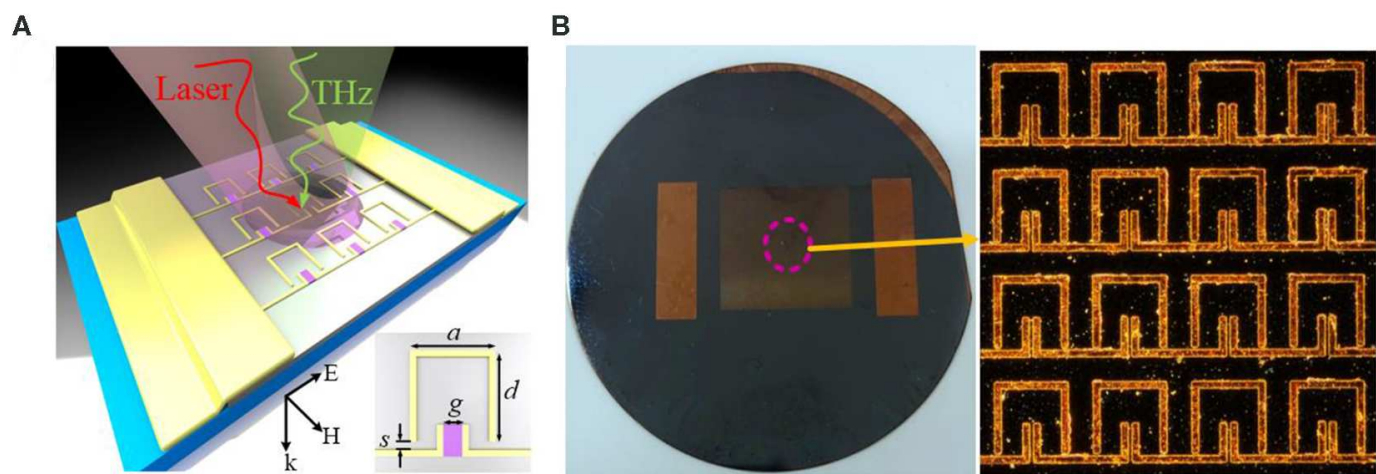

FIGURE 1 | (A) Three-dimensional schematic diagram of the proposed metasurface embedded with perovskite quantum dots (CsPbBr ${ }_{3}$-QDs). (B) Optical microscope image of the fabricated $\mathrm{CsPbBr}_{3}-\mathrm{QD}$-embedded metasurface (geometric parameters of the metasurface unit cell, $a=75 \mu \mathrm{m}, \mathrm{g}=12 \mu \mathrm{m}, \mathrm{s}=12 \mu \mathrm{m}$, and $d=70 \mu \mathrm{m})$. 


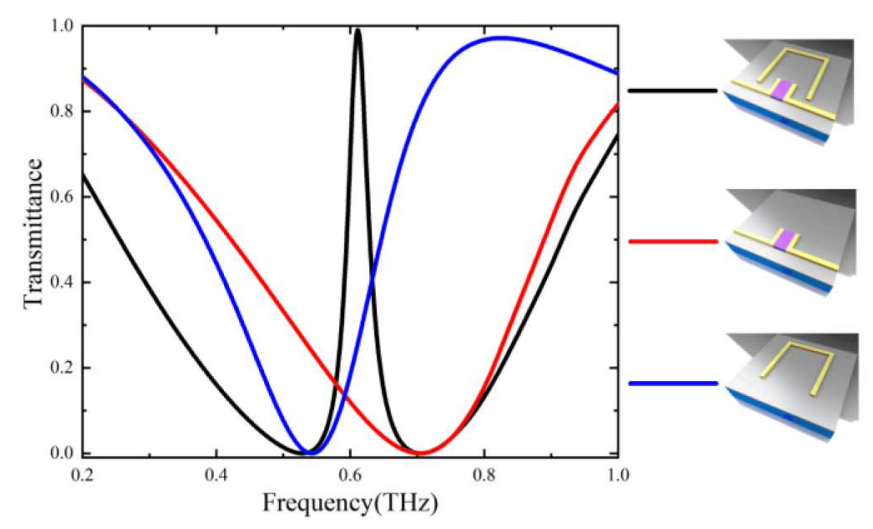

FIGURE 2 | Terahertz transmission spectra of the different parts of the proposed metasurface unit cell.

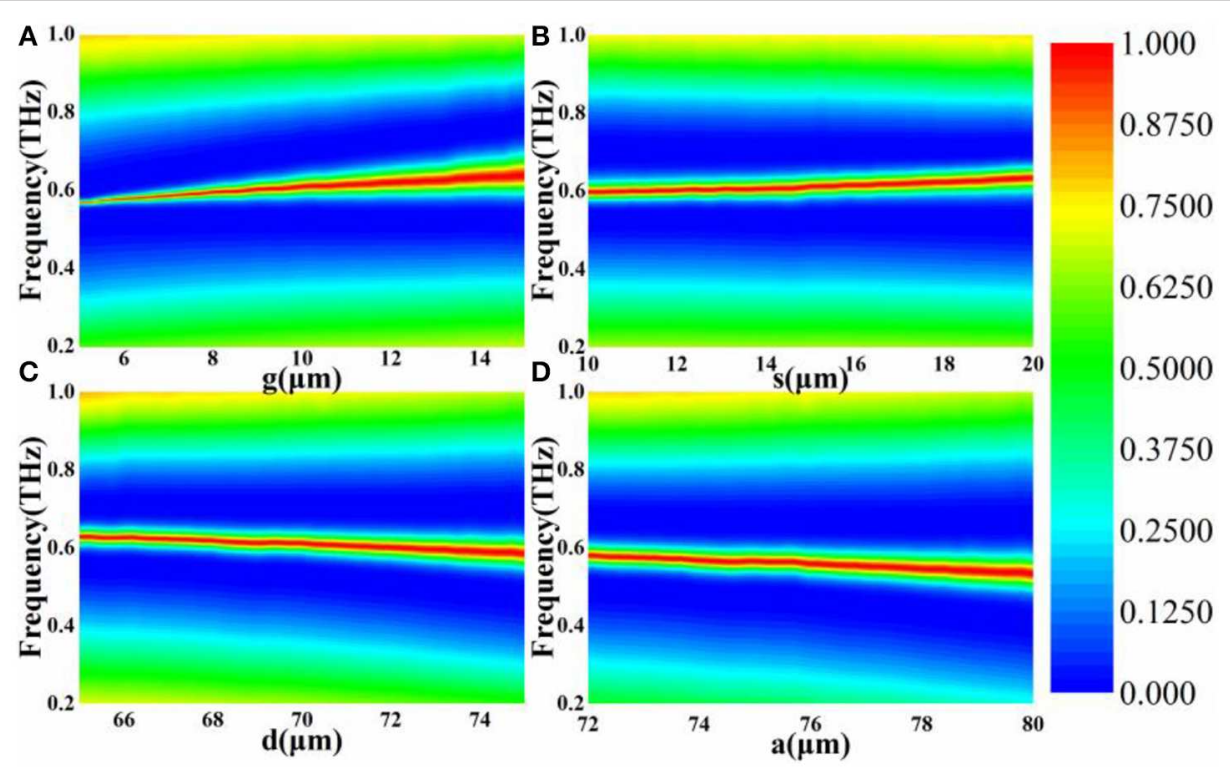

FIGURE 3 | Terahertz transmittance contour plot for various geometrical parameters values of our proposed $\mathrm{CsPbBr}_{3}-\mathrm{QD}$-embedded metasurface (A) $g$, (B) $s$, (C) $d$, (D) $a$.

with a different power density. The excitation source was a Ti:sapphire laser with $100-\mathrm{fs}$ duration at $80-\mathrm{MHz}$ repetition rate, and working wavelength at $780 \mathrm{~nm}$. Terahertz pulse was generated using LT-GaAs photoconductive antenna, and a $\mathrm{ZnTe}$ nonlinear crystal was used to detect the terahertz signal. The $\mathrm{CsPbBr}_{3}$-QD-embedded metasurfaces were placed at the confocal position of the system. The entire experiment was carried out in nitrogen environment. The recorded terahertz transmission time domain spectra are shown in Figure 4A, for varying the $\mathrm{CW}$ pumping laser fluence. Figure 4B shows the terahertz frequency domain spectra by Fourier transformation from the time domain data under various laser pump powers. In Figure 4, one can see that the transmission spectra of the $\mathrm{CsPbBr}_{3}$-QD-embedded metasurface declines gradually as the pumping laser power increases. When the pumping power increases to $240 \mu \mathrm{J} / \mathrm{cm}^{2}$, the transmittances of the terahertz wave drops to $10.9 \%$ at $0.5 \mathrm{THz}$. It indicates that the sample can control the terahertz wave transmission under a different pump laser power.

The terahertz transmission switching performance of our proposed structure was investigated in terahertz continuous wave system (see Figure 5). Figure 6 plots the measured dynamic characteristics of the proposed $\mathrm{CsPbr}_{3}$-QD-embedded metasurface structure with various laser pump fluences. Figures 6A,B illustrate the detected voltage signal waveform shape for switching speeds of $10 \mathrm{KHz}$ and $8 \mathrm{MHz}$. One can see that the transmission amplitude of terahertz wave decreases with the switching speed increase from $10 \mathrm{KHz}$ to $8 \mathrm{MHz}$. 

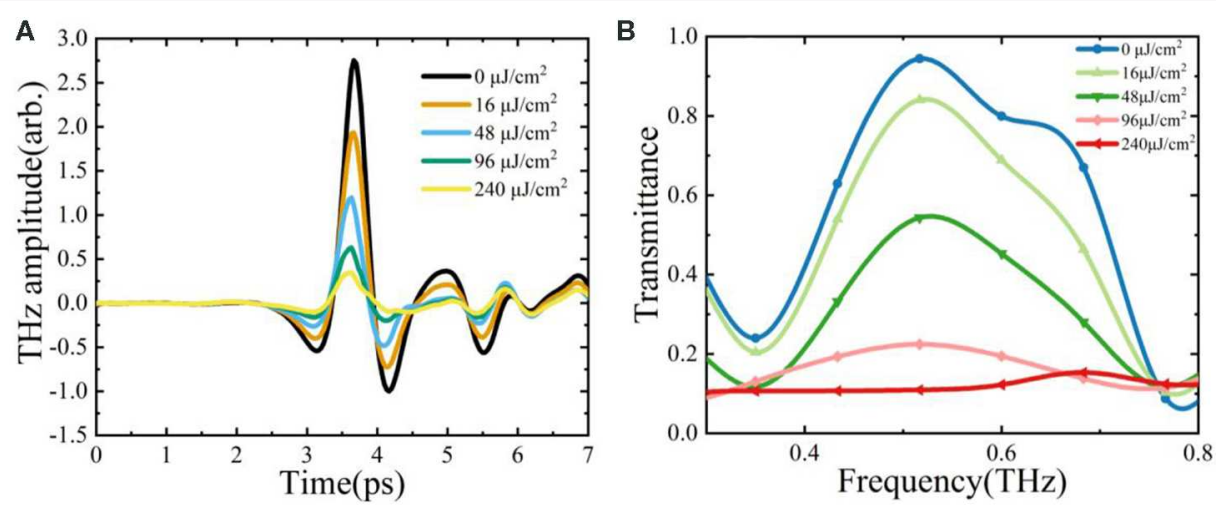

FIGURE 4 | (A) Measured terahertz transmission time domain spectra using the terahertz time domain spectroscopy (THz-TDS) under a different laser power. (B) Corresponding terahertz frequency domain spectra.

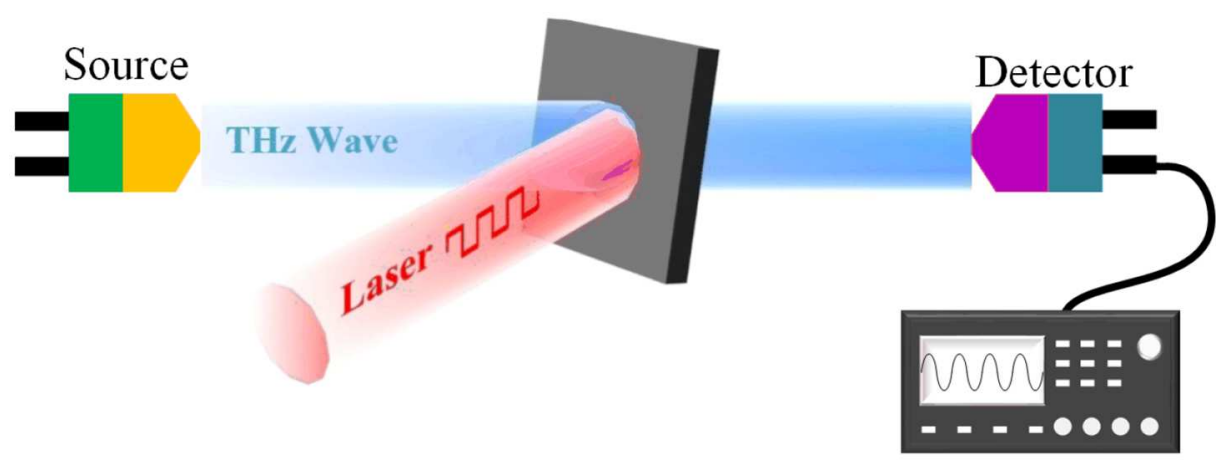

FIGURE 5 | Dynamic switching measurement using a backward-wave oscillator (BWO) CW terahertz source and Schottky diode detector.
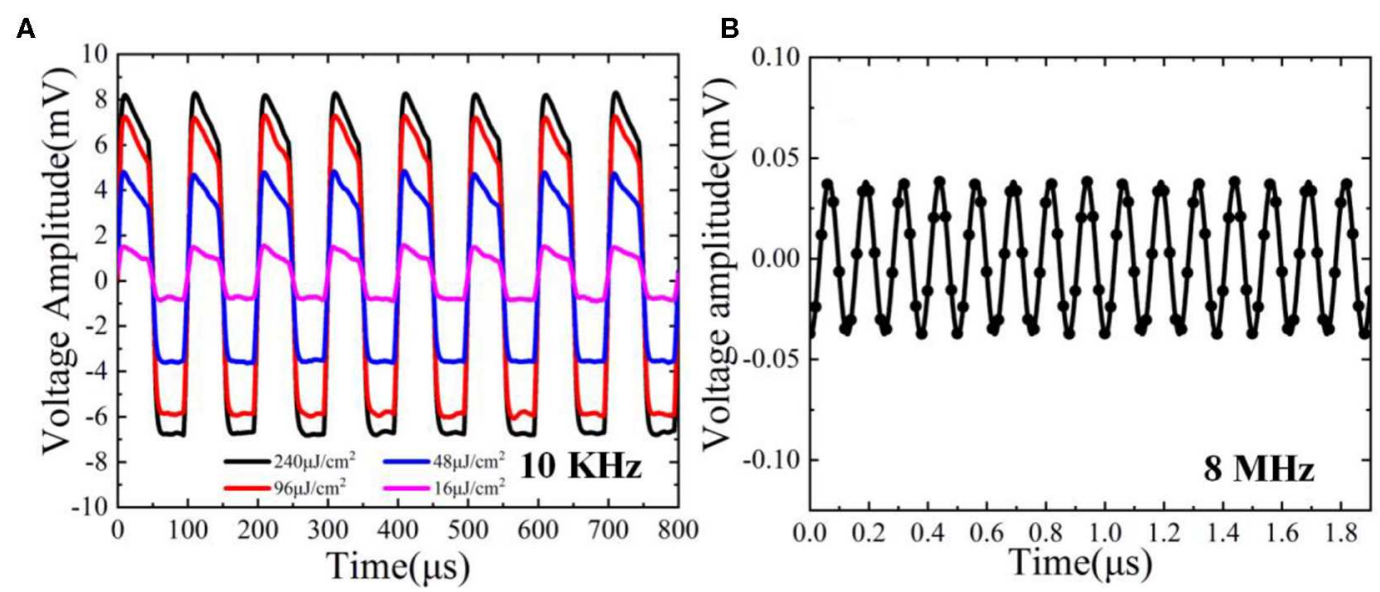

FIGURE 6 | (A) A 10-KHz switching speed under different pump laser fluencies. (B) An 8-MHz switching speed of the sample.

At switching speed of $8 \mathrm{MHz}$, the detected voltage amplitude falls to $0.05 \mathrm{mV}$. Figure $7 \mathrm{~A}$ shows the terahertz transmission intensity distribution through the $\mathrm{CsPbr}_{3}-\mathrm{QD}$-embedded metasurface structure without pump laser fluence. The terahertz wave transmission intensity drops to $10.9 \%$ of its original value under $240 \mu \mathrm{J} / \mathrm{cm}^{2}$ pump laser intensity. As depicted in Figure $\mathbf{7 B}$, the corresponding extinction ratio is $83 \%$. Figure $7 \mathbf{C}$ shows the extinction ratio dependence of the pump laser fluence 


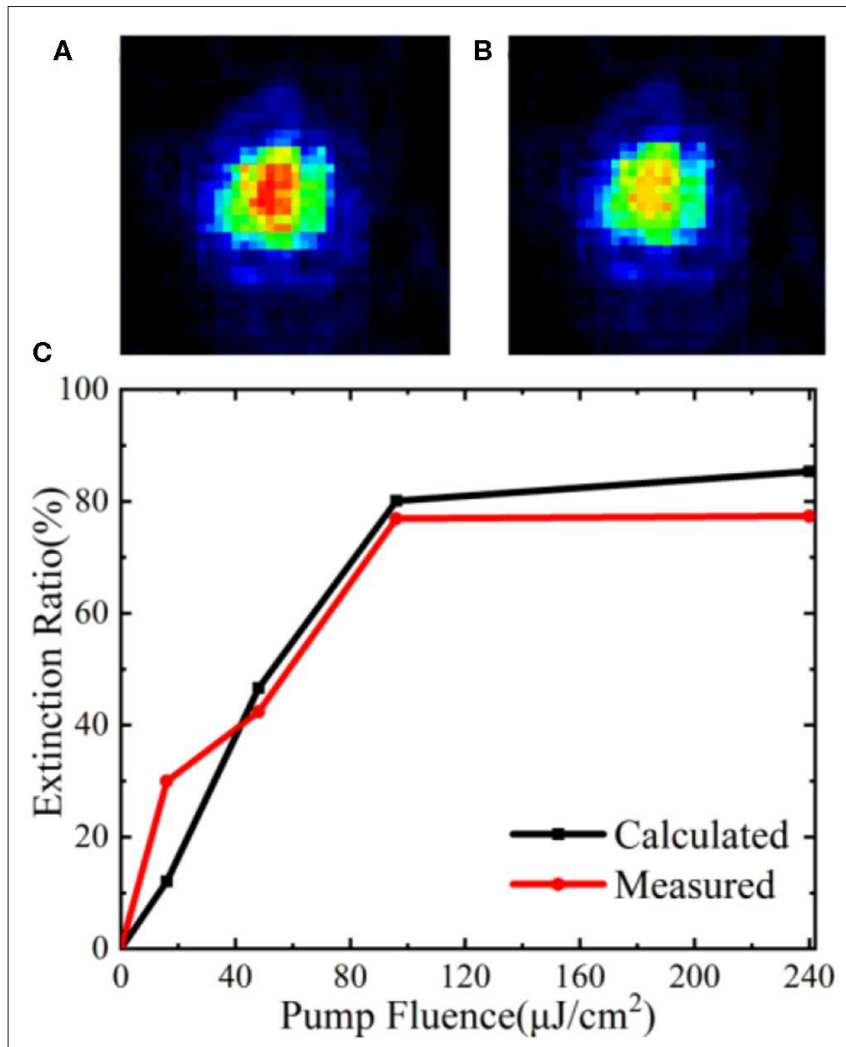

FIGURE 7 | Terahertz intensity distributions through the $\mathrm{CsPbBr}_{3}-\mathrm{QD}$-embedded metasurface. (A) $0 \mu \mathrm{J} / \mathrm{cm}^{2}$ and (B) $240 \mu \mathrm{J} / \mathrm{cm}^{2}$ pump laser intensity. (C) Extinction ratio dependence of pump laser intensity.

obtained by experiment and calculation. One sees that the $\mathrm{Cs} \mathrm{PbBr}_{3}$-QD-embedded metasurface has low saturation pump laser intensity and high extinction ratio factor as a potential method to actively control the terahertz wave transmission. This work demonstrates a new approach for realizing active terahertz devices with improved functionalities.

\section{CONCLUSION}

We describe a method for the active control of terahertz wave transmission using $\mathrm{CsPbBr}_{3}$-QD-embedded metasurface. The experimental results confirm the numerically simulated expectations. With the external applied pump laser irradiation, our presented terahertz device achieves highefficiency terahertz wave switch with an off-on speed of $8 \mathrm{MHz}$. Owing to effective switching and easy fabrication, this device has promising applications as a controllable switch in future terahertz wave communication and imaging systems.

\section{DATA AVAILABILITY STATEMENT}

All datasets generated for this study are included in the article/supplementary material.

\section{AUTHOR CONTRIBUTIONS}

R-HX performed the $\mathrm{THz}$ measurements and did the calculations. X-QP fabricated and characterized the devices. J-SL concerned the devices structure and developed the theoretical model and guided the experimental work. All authors discussed the results and co-wrote the manuscript.

\section{ACKNOWLEDGMENTS}

The authors acknowledge valuable discussion on experiment with Dr. J. Liu. This work was sponsored by the National Natural Science Foundation of China $(61871355,61831012)$ and Zhejiang Lab (NO.2019LC0AB03).

\section{REFERENCES}

1. Chan W, Deibel J, Mittleman D. Imaging with terahertz radiation. Report Progr Phys. (2007) 70:1325. doi: 10.1088/0034-4885/70/8/R02

2. Koenig S. Wireless sub-THz communication system with high data rate. Nat Photon. (2013) 7:977. doi: 10.1038/nphoton.2013.275

3. Han $P$, Cho G, Zhang X. Time-domain transillumination of biological tissues with terahertz pulses. Opt Lett. (2000) 25:242. doi: 10.1364/OL.25.000242

4. Savo S, Shrekenhamer D, Padilla W. Liquid crystal metamaterial absorber spatial light modulator for THz applications. Adv Opt Mater. (2014) 2:2759. doi: 10.1002/adom.201300384

5. Wang J, Tian H, Wang Y, Li X, Cao Y, Li L, et al. Liquid crystal terahertz modulator with plasmon-induced transparency metamaterial. Opt Exp. (2018) 26:5769-76. doi: 10.1364/OE.26.005769

6. Sensale-Rodriguez B, Yan R, Kelly M. Broadband graphene terahertz modulators enabled by intraband transitions. Nat Commun. (2012) 3:780. doi: $10.1038 /$ ncomms 1787

7. Tabatabaei F, Biabanifard M, Abrishamian M. Terahertz polarizationinsensitive and all-optical tunable filter using Kerr effect in graphene disks arrays. Optik. (2019) 180:526 doi: 10.1016/j.ijleo.2018.11.103

8. Yu T, Chi N, Tsai H, Wang S, Luo C, Chen K. Robust terahertz polarizers with high transmittance at selected frequencies through Si wafer bonding technologies. Opt Lett. (2017) 42:4917 doi: 10.1364/OL.42.004917

9. Reichel K, Mendis R, Mittleman D. A broadband terahertz waveguide T-junction variable power splitter. Sci Rep. (2016) 6:28925 doi: 10.1038/srep28925

10. Hochberg M, Baehrjones T, Wang G, Shearn M, Harvard K, Luo J. Terahertz all-optical modulation in a silicon-polymer hybrid system. Nat Mater. (2006) 5:703-9 doi: 10.1038/nmat1719

11. Grant J, Ma Y, Saha S, Lok L, Khalid A, Cumming D. Polarization insensitive, broadband terahertz metamaterial absorber. Opt Lett. (2011) 36:3476-8 doi: 10.1364/OL.36.003476

12. Kupchak C, Erskine J, England D, Sussman B. Terahertzbandwidth switching of heralded single photons. Opt Lett. (2019) 44:1427 doi: 10.1364/OL.44.001427

13. Li J, He J, Hong Z. Terahertz wave switch based on silicon photonic crystals. Appl Opt. (2007) 46:5034-7. doi: 10.1364/AO.46.005034

14. Chen S, Fan F, Miao Y, He X, Zhang K, Chang S. Ultrasensitive terahertz modulation by silicon-grown $\mathrm{MoS}_{2}$ nanosheets. Nanoscale. (2016) 8:4713-9. doi: 10.1039/C5NR08101G 
15. Zheng W, Fan F, Chen M, Chen S, Chang S. Optically pumped terahertz wave modulation in $\mathrm{MoS}_{2}-\mathrm{Si}$ heterostructure metasurface. AIP $A d v$. (2016) 6:075105. doi: 10.1063/1.4958878

16. Liu W, Fan F, Xu S, Chen M, Wang X, Chang S. Terahertz wave modulation enhanced by laser processed PVA film on Si substrate. Sci Rep. (2018) 8:8304. doi: 10.1038/s41598-018-26778-7

17. Luo L, Wang K, Ge C, Guo K, Shen F, Yin Z, et al. Actively controllable terahertz switches with graphene-based nongroove gratings. Photonics Res. (2017) 5:604. doi: 10.1364/PRJ.5.000604

18. Snaith H. Perovskites: the emergence of a new era for low-cost, high-efficiency solar cells. J Phys Chem Lett. (2013) 4:3623-30. doi: 10.1021/jz4020162

19. Green M, Ho-Baillie A, Snaith H. The emergence of perovskite solar cells. Nat Photon. (2014) 8:506-14. doi: 10.1038/nphoton.2014.134

20. Stranks S, Eperon G, Grancini G, Menelaou C, Alcocer M, Leijtens $\mathrm{T}$, et al. Electron-hole diffusion lengths exceeding 1 micrometer in an organometal trihalide perovskite absorber. Science. (2013) 342:3414. doi: $10.1126 /$ science. 1243982

21. Herz L. Charge-carrier dynamics in organic-inorganic metal halide perovskites. Annu Rev Phys Chem. (2016) 67:6589. doi: 10.1146/annurev-physchem-040215-112222
22. Yettapu G, Talukdar D, Sarkar S, Swarnkar A, Nag A, Ghosh P. $\mathrm{THz}$ conductivity within colloidal $\mathrm{CsPbr}_{3}$ perovskite nanocrystals: remarkably high carrier mobilities and large diffusion lengths. Nano Lett. (2016) 16:4838. doi: 10.1021/acs.nanolett.6b 01168

23. Chanana A, Liu X, Zhang C, Vardeny Z, Nahata A. Ultrafast frequencyagile terahertz devices using methylammonium lead halide perovskites. Sci Adv. (2018) 4:7353. doi: 10.1146/annurev-physchem-040215112222

Conflict of Interest: The authors declare that the research was conducted in the absence of any commercial or financial relationships that could be construed as a potential conflict of interest.

Copyright (C) 2020 Xiong, Peng and Li. This is an open-access article distributed under the terms of the Creative Commons Attribution License (CC BY). The use, distribution or reproduction in other forums is permitted, provided the original author(s) and the copyright owner(s) are credited and that the original publication in this journal is cited, in accordance with accepted academic practice. No use, distribution or reproduction is permitted which does not comply with these terms. 